\title{
Towards True Process Descriptions Interoperability
}

\author{
Tomislav Rozman, Romana Vajde Horvat and Gregor Polančič \\ Institute of Informatics, Faculty of Electrical Engineering and Computer Science, University of Maribor, Slovenia
}

\begin{abstract}
The article describes a part of our research in the area of (business) process modeling, analysis and execution. It describes the current state of process languages and standards. It concentrates on the process model interoperability and portability problems, caused because of huge number of process modeling standards, which often do not even target the same conceptual level of process modeling. It provides an idea how to bring together those standards, using process concepts mapping into Petri nets and incorporating the usage of process patterns. Transformation to strictly formal process languages has a potential to prove itself as a fair solution with the following advantages: the number of mappings among different process languages is reduced and the process model can be analyzed using proven Petri Net or general graph algorithms. Development of the approach is still in its early phase. Mapping rules to/from process patterns are neither strictly defined nor trivial, therefore, they will be the main focus of our future research.
\end{abstract}

Keywords: process modeling, process analysis, transformation, Petri nets, BPM.

\section{Introduction}

A business process engineer is often confronted with the dilemma, which 'complete, all-in-one process management solution' to choose, that fits his/her organization best. After a research (crawling through the jungle of marketing materials and flashy presentations), he finds out there are some nearly complete, but specific solutions. In fact, some of these so called 'business process management suites' are often legacy products, wrapped in new disguise. On the other hand, there is a great number of partial solutions. Those solutions are often intended for different audiences; therefore they often do not share the same conceptual level. Some of these solutions are intended only for highlevel graphical process modeling, other only for machine-level process model interchange and execution. Almost the same situation can be noticed in standard process modeling languages. It does not matter which solution is chosen, the result is, the process models created in specific environment are vendor-dependent and cannot be simply plugged in different vendor's process management suite.

Some years ago, there were only a few standards for workflow description. The most promising one was developed by WFMC (WorkFlow Management Coalition) as a result of the incompatible workflow products of that time, but none of them had really fully supported it. Newer standards, based on XML, are promising, but their wider adoption is hindered due to a great number of such languages [10].

Current development and research in the BPM area is focused on separating the process definition from its execution environment. This approach presumes there is only one universal process description language, which can be interpreted by many process environments. But, at the time of writing of this paper, there are several competing standards, for example, BPML (Business Process Modeling Language), BPEL (Business Process Execution Language), XPDL (XML Process Definition Language), ebXML (Electronic Business XML Initiative).

Nowadays, a lot of researchers and organizations invent their own languages, standards or extensions to existing process modeling languages. There are several mainstream solutions for process description, which are under heavy evolution process.

Based on experiences from other research areas dealing with mapping of the real world 'things' 
to conceptual models, it is not likely that allsatisfying process modeling language will emerge.

Therefore, transformations among different process modeling languages must exist in order to keep compatibility among them. Because of great number of process description languages, a lot of mapping rules should exist too.

Therefore, we propose the solution which reduces the number of mappings among different process modeling languages.

\section{Paper Organization}

The rest of the paper is organized as follows: first, related work in this research area is summarized. Next, the basic idea of proposed approach is presented, which encompasses the rationale for the research and briefly describes the proposed architecture. Further, the core elements of the architecture (process patterns mapping modules and workflow patterns) are explained. Possible applications of the proposed architecture are discussed.

Last chapters end with some future research directions and ideas.

\section{Related Work}

A huge number of today's partially compatible process description languages increase the portability problems among them, as reported by many authors [7], [9], [10].

An effort to make a common process language is held by [10], that results in the development of the PSL (Process Specification Language), which has evolved from the PIF (Process Interchange Format) and KIF (Knowledge Interchange Format). PSL is also in the process of standardization, as an ISO/IEC 18629 standard [12]. PSL ontology is based on situationcalculus and consists of the following basic elements: activity, activity-occurrence, time-point and object, which is also the reason, why some authors [8] argue about suitability of PSL as the common process language. They propose Petri nets and pi-calculus as basics for the foundation language, mainly because today's process languages are based on message passing mechanisms and not on the time constraints. To make the situation more confusing, it is possible to transform PSL-based process model into Petri nets [11].

An effort to make Petri nets more useful for workflow modeling is held by [3]. Research group of Department of Technology Management, Eindhoven University of Technology made a big contribution to characterization and analysis of modern, XML-based business process modeling languages, using workflow patterns. Static structure of those patterns can be described using WF-nets, which were introduced by [3].

The study of several mappings among process description languages was made, using specifications [13], [14], [15] and others.

\section{Research Method}

At this stage, the non-reactive method has been selected, because the proposed approach is under development and the idea is still evolving. The existing approaches are studied, compared and combined.

\section{Basic Idea}

As was mentioned earlier, there is a problem about the number of mappings among different process modeling languages. If we want full interoperability among all the process description languages, there are $n(n-1)$ mappings [9] in fully connected graph [Figure 1], where the nodes PLx represent process language, the arcs

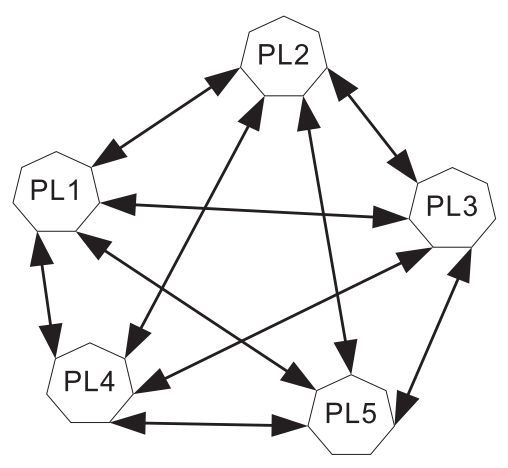

Fig. 1. The graph of mappings among process modeling languages. 
represent two-way mappings and the $n$ is the number of nodes.

If the languages are XML-based, the mapping among them becomes easier, because of the possibility of the XSLT transformations usage, but their number stays the same. The problems arise if the process description languages are not directly comparable, for example, if they cover different process modeling aspects. Therefore, some conditions need to be satisfied when transforming the process description from (PL1) to (PL2):

- Between the PL1 and the PL2 there must be a 'path', or sequence of defined mapping rules.

- Process concept from every process description language on this path must be covered in its predecessor.

Fortunately, that is the worst-case scenario.

Ideally, only one common ('core') process modeling language should exist, encompassing all of the possible process modeling concepts of all languages. In this ideal situation, the number of mappings is only $2^{*} n$, where $n$ represents the number of process languages. All mappings among process description languages are indirect; to transform the process description from language (PL1) to (PL2), the intermediate transformation to the 'core' language (CPL) is needed.



Fig. 2. Graph of mappings (one common process description language).

To realize this idea, the following conditions should be satisfied:

- A core language for the process descriptions should exist, with strong mathematical foundations and

- Two-way mappings to convert to and from the core process language should be defined.
In reality, some mainstream process modeling languages are evolving. The graph of mappings is also neither fully connected, nor bidirectional, which means that only a small set of mappings exists today. For example:

- BPMN (Business Process Modeling Notation) $\rightarrow$ BPML/BPEL [13],[14],

- XRL (eXtensible Routing Language) $\rightarrow$ PNML (Petri Net Markup Language).

Those process description languages, 'convergence points' or, de-facto standards, reduce the number of mappings needed. The number of mappings here is between the positive and the negative scenarios.

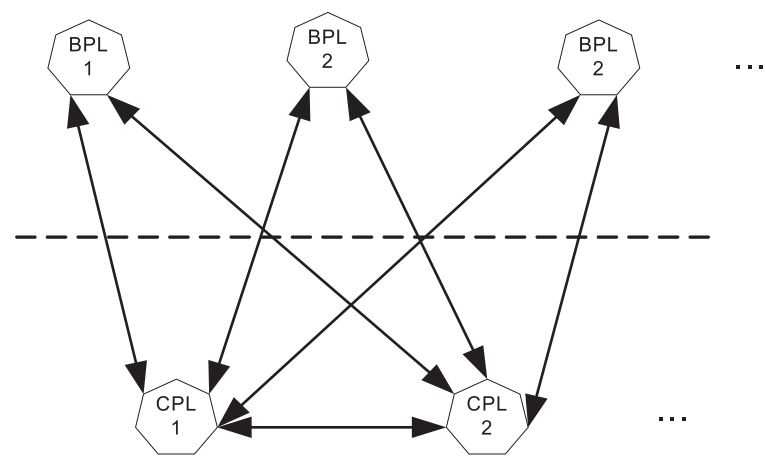

Fig. 3. Leveled graph of mappings among process modeling languages.

Such topology is 'right' only if the core process description languages are compatible with one another, i. e. if they are derived from common low-level process language.

Relying on the current state of the development results in this field, majority of the parts of the proposed architecture and the stack of mappings [Figure 4] already exist. What is still missing, are the model, mappings and relations, which connect high-level and low-level process description languages and formally define mapping rules.

The proposed architecture contains the following tiers:

- The top-level tier, which consists of current and future process description languages, with only limited set of defined mappings among one another, and 


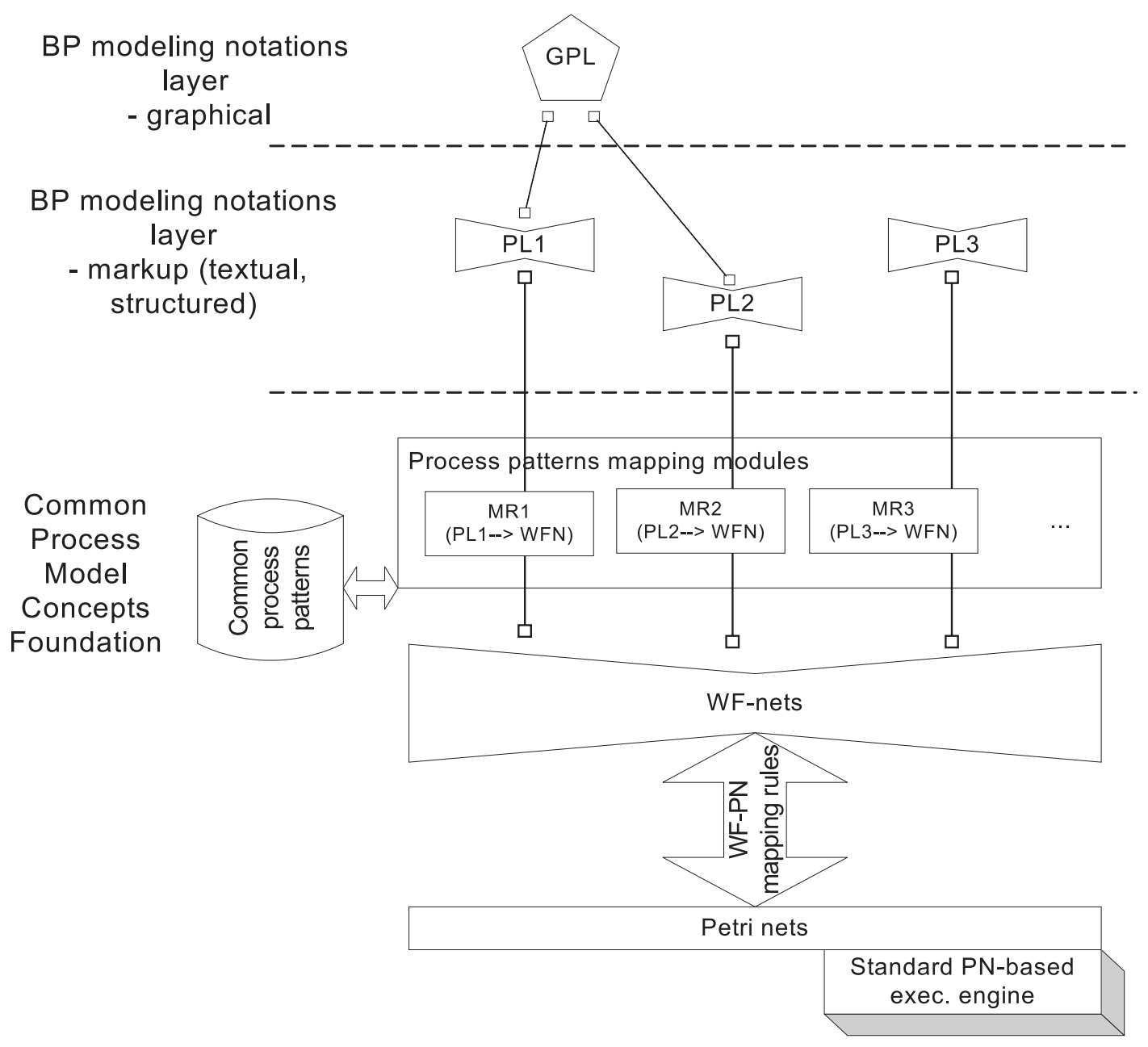

Fig. 4. Proposed mapping architecture.

- Common Process Model Concepts Foundation layer, which consists of WF-net and Petri net layers. On top of the WF-net layer is the most important part, the mapping layer, which converts variety of process description languages to WF-nets.

For the core process description language, the Petri nets were chosen, because their advantages for a workflow modeling were proven many times.

WF-nets were chosen, because an extensive analysis showed they are appropriate for process and workflow representation [3]. Main reasons are:

- WF-nets are high-level Petri nets, and they can be converted to basic Petri nets,

- WF-nets are more appropriate for a workflow modeling than basic Petri nets, because they overcome their limitations (advanced routing and triggering constructs),

- WF-nets are Turing-complete, similar as other high-level Petri-nets, which means that any algorithm can be expressed and,

- WF-nets are very appropriate for formal notation of workflow patterns.

The core of the process pattern-mapping modules [Figure 4] is the WF-pattern with several mapping rules defined.

\section{Structure of the Pattern-Mapping Modules}

The kernel of the pattern-mapping module [Figure 5] is the WF-pattern. Every WF-pattern can be represented with the WF-net, which can be 


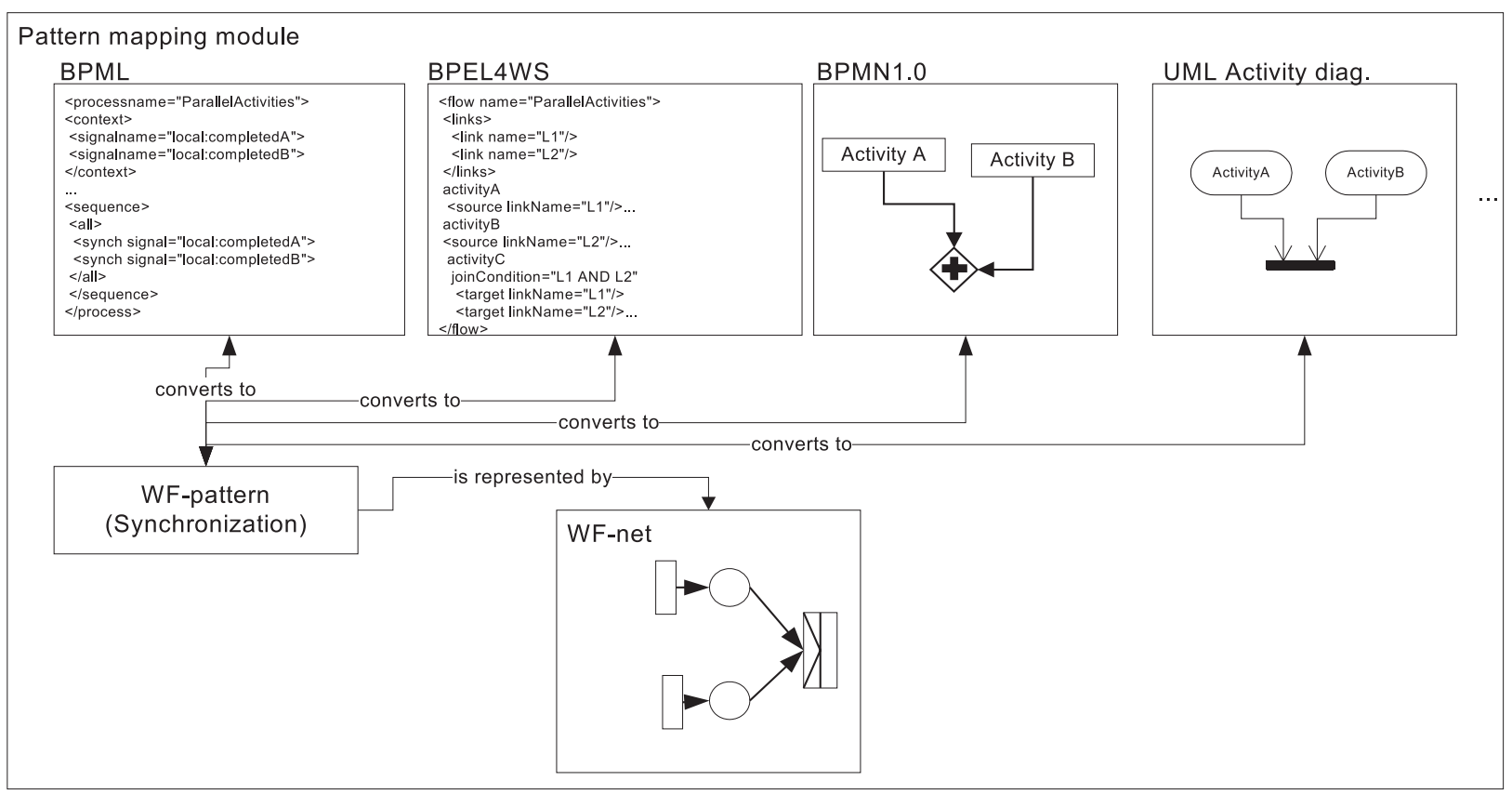

Fig. 5. Example of mapping rule for Synchronization pattern.

transformed into Petri net. The mapping module contains mapping rules for conversion to the specific process description languages.

The pattern-mapping module is extensible with additional mapping rules. An example of such pattern-mapping module is presented in [Figure 5]. The example pattern mapping module describes how the pattern synchronization is presented in several process description languages: graphical (BPMN and UML Activity diagrams) and one markup language (BPML). The synchronization pattern represents a point in the process where multiple parallel branches converge into one single thread of control.

Examples of the mappings from synchronization to BPML and other WF-patterns are described in detail in [4]. Other mappings are described in [5] (BPEL), [6] (XPDL), as a result of the pattern-based analysis of the web services composition languages.

WF-nets describe only static structure of the pattern-mapping module. For the definition of the WF-net semantics, different approaches are studied, for example, the PSL [11].

WF-nets and Petri nets do not have a mechanism to describe information objects which are very important in a process modeling. Therefore, the usage of RDF (Resource Definition Framework) for a detailed token description is studied [9].
Because of the importance of WF-patterns in our approach, the following paragraph briefly describes them.

\section{WF-Patterns}

The pattern is the abstraction from a concrete form which keeps recurring in specific nonarbitrary contexts [2]. WF-pattern (Workflow pattern) is a combination of interconnected process modeling concepts, which formulate routing constructs, different synchronization or communication mechanism.

In the paper [3], the following groups of WFpatterns are identified:

1. Basic control flow patterns, which are the basic constructs present in most workflow and process description languages. They are used for sequential, parallel and conditional routing (Sequence, Parallel Split, Synchronization, Exclusive Choice, Simple Merge).

2. Advanced branching and synchronization patterns describe advanced types of splitting and joining behavior (Multi-choice, Synchronizing Merge, Multi-merge, Discriminator).

3. Structural patterns are block structures that define workflow entry and exit points. In 
graphical languages, block structures are often considered to be too restrictive. Therefore, the patterns were identified that allow a less rigid structure (Arbitrary Cycles, Implicit Termination).

4. Patterns involving multiple instances describe parts of the process which need to be instantiated several times (Multiple Instances Without Synchronization, Multiple Instances With a Priori Design Time Knowledge, Multiple Instances With a Priori Runtime Knowledge, Multiple Instances Without a Priori Runtime Knowledge).

5. State-based patterns describe state-based behavior of the process or workflow (Deferred Choice, Interleaved Parallel Routing, Milestone).

6. Cancellation patterns describe, how the process is canceled, based on incoming events and what are the steps following cancellation (Cancel Activity, Cancel Case).

WF-patterns are conceptual constructs and they are not well covered in majority of process definition languages. This could lead to mapping problems and the specific process information can be lost. The following paragraph discusses possible work-around solutions.

\section{The Specific Process Model Enhancements Problem}

Unfortunately, some process constructs are language specific and cannot be mapped to another process description language. On the other hand, some workflow patterns do not have direct representation in currently available process description languages. Sometimes, workaround solutions can be used. For example [4], BPML does not support Multichoice, Discriminator or Synchronization merge pattern directly, although this pattern can be expressed using combination of simpler concepts.

If we want to accomplish true process descriptions interoperability, all vendor specific process information should be preserved. This information should be hidden and marked 'inactive' if the process model is mapped to the language which does not support it.

Similar approach is used in some HTML codegeneration tools. They create generic code and additional specific information, which are hidden in comments and ignored by web browsers. Another example of information hiding is $<$ head $>$ part of HTML code. The $<$ head $>$ part is not rendered by the web browser (except for the $<$ title $><$ /title $>$ element), although it contains essential information for different use cases (web spiders and search engines).

\section{Possible Applications}

Practical usage of presented approach could be a web-based service of a process model repository and a translator. A user wishing to transform his process model would 'feed' the service with the process model and desired destination format. The service would check the model, transform it and return it to the user.

\section{Discussion}

Benefits of such architecture are: the process definition (model) could be independent, not only from runtime (or, simulation environment), but also from the process language.

The main result of this approach is: (a) it will not matter any more in which process language the process is described and (b) the process models could be analyzed using proven Petri net or graph algorithms.

The idea is partly borrowed from the programming language evolution: first, there were hardware specific programming languages, then operating system specific languages and lastly, they developed into the problem area specific languages. Last ones can run on different platforms, because the source code is not compiled directly into a hardware and OS specific binary code, but into an intermediate level byte code, which is interpreted by virtual machine. Main programming language vendors have adopted this approach: Sun with Java and Microsoft with C\#.

It was recognized [1] that the closer the syntax, rules, and mnemonics of the programming language could be to "natural language", the less likely it became that the programmer would inadvertently introduce it into the program. Similar thinking could be applied to process modeling languages. 


\section{Conclusion}

We do not claim the proposed approach is exhaustive or optimal. It is a hybrid of multiple existing efforts trying to create universal process description and interchange language. It tries to take the advantages out of them.

At the moment, the proposed approach is still at a draft stage.

Our future work will concentrate on further development of the suggested approach: the mapping modules will be defined in detail, refined and tested on practical, real-world examples.

\section{References}

[1] Evolution of High-Level Languages, http://www.encyclopedia.com/html/ section/progrlanEvolutionof HighLevelLanguages . asp [10/20/2003].

[2] E. Gamma, R. Helm, R. Johnson, and J. VlisSIDES, Design Patterns: Elements of Reusable Object-Oriented Software. Professional Computing Series, Addison Wesley, Reading, MA, USA, 1995.

[3] WIL M. P. VAN DER AALST ET AL, Workflow Patterns: On the Expressive Power of (Petri-net-based) Workflow Languages, http: //tmitwww. tm. tue. $\mathrm{nl} / \mathrm{research} /$ patterns/download/ invited_talk_cpn_2002.pdf [01/10/2004].

[4] WIL M. P. VAN DER AALST ET AL, Pattern-Based Analysis of BPML (and WSCI), http: // tmitwww.tm.tue.nl/research/patterns/ download/qut_bpml_rep.pdf [01/10/2004].

[5] WIL M. P. VAN DER AALST ET AL, Pattern-Based Analysis of BPEL4WS, http://tmitwww.tm. tue.nl/research/patterns/download/ qut_bpel_rep.pdf [01/10/2004].

[6] WIL M. P. VAN DER AALST, Patterns and XPDL: A Critical Evaluation of the XML Process Definition Language, http://tmitwww.tm.tue. $\mathrm{nl} / \mathrm{research} /$ patterns/download/ ce-xpdl.pdf [01/10/2004].

[7] BPM\&BIM,http://www.cbop.gr.jp/dl/semi /bosc/2001/011102bosc_05.pdf [03/01/2004].

[8] JoHN F. SowA, The WonderWeb Foundational Ontology Library and the DOLCE Ontology, http://suo. ieee.org/email/msg08943.html [13/01/2004].

[9] JoshuA Lubell, XML Representation of Process Descriptions, http://ats.nist.gov/psl/xml/ process-descriptions . html [3/12/2003].
[10] SHARON BoyeS-Schiller, Putting Workflow and BPM Standards into Context, e-Science Workflow Services, Edinburgh, [03/12/2003], http: www.nesc.ac.uk/talks/303/ EdinburghPresentation2122003.pdf [02/02/2004].

[11] PSL home page, http://www.mel.nist.gov/ psl/index.html [02/02/2004].

[12] ISO TC184/SC4, Setting the Standards for Industrial Data Homepage, http: //www.tc184-sc4.org/ [02/02/2004].

[13] BPMI.org, Business Process Modeling Notation, Working Draft (0.9), November 13, 2002, http: //www.bpmi.org/bpmn-spec.esp [20/12/2002].

[14] BPMI.org, Business Process Modeling Notation, (1.0), http: //www . bpmi.org/bpmn-spec . esp [25/09/2003].

[15] ASSAF ARKIN, Intalio, Business Process Modeling Language, http://www.bpmi.org/bpml-spec . esp [22/07/2002].

Received: June, 2004 Accepted: June, 2004

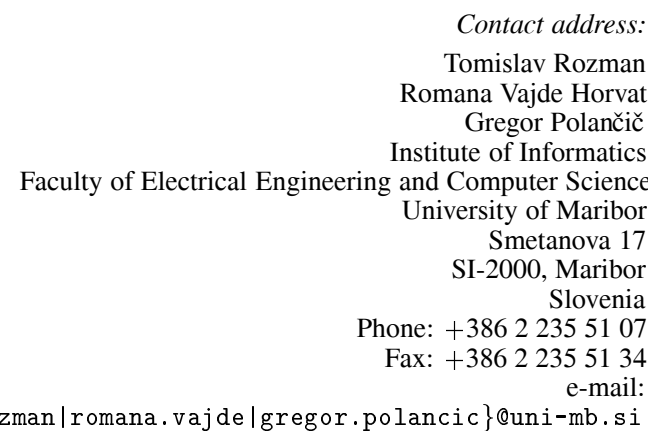

\{tomi.rozman|romana.vajdelgregor.polancic\}@uni-mb.si

TOMISLAV ROZMAN is a PhD student, teaching assistant and research engineer at the Faculty of Electrical Engineering and Computer Science, University of Maribor. His work and research interests are dedicated to software standards, process and workflow modeling and analysis and web application development

ROMANA VAJDE HORVAT, $\mathrm{PhD}$, is an assistant professor at the Faculty of Electrical Engineering and Computer Science, University of Maribor She teaches and works in the field of software process improvement, software quality improvement, project management, information society and computer-mediated communication. She is also head of the Centre of Information Technology, Maribor

GREGOR POLANČIČ is a PhD student at the Faculty of Electrical Engineering and Computer Science, University of Maribor. He is a teaching assistant of computer science. His research work is focused on the development of web applications based on the frameworks and the development of open source software. 